\title{
Three-Dimensional Aberration-Free Imaging Recovery by the Oversampling Method and Tomography
}

\author{
Wan-Yi Yen, Fu-Ron Chen and Ji-Jung Kai \\ Center for Electron Microscopy, Department of Engineering and System Science, \\ National Tsing-Hua University, Hsinchu, Taiwan. 30043, R.O.C.
}

As the electrons passing through the lens systems in a TEM, the unavoidable lens aberrations blur the image and distorted the phase of the image wave such that the recorded images do not actually reflect the true structure. Although, the lens aberrations do not affect the intensity of diffraction beam, the phase information in the diffraction plane lost during the recording procedure. The phase in reciprocal space relates to the relative position of atoms in the sample. That is, by solving the phase problem in the reciprocal space, the aberration-free imaging can be recovered.

In 1972, Gerchberg and Saxton [1] proposed that by iterative algorithm method one is able to retrieve the phase in real and reciprocal space, basing on the measurements of intensities of the image and the diffraction patterns. As the time going Fienup in 1982 adds the feedback [2], who supported some constraint in real and reciprocal space to G-S method. This process is shown simply in Figure 1. Only the intensity of diffraction patterns and the shape of the object are needed. And one should notice that before the iteration the sampling in reciprocal space needed to be checked if all the information of intensity was not got lost [3]. During the iteration a renew phase is gained under the fixed intensities in reciprocal space and the constraint in real space.

The $\mathrm{MgO}$ was selected for demonstration because it's light element compositions and well known structure. We simulate the diffraction intensities of an $\mathrm{MgO}$ nano-particle which contains 27 unit cells in JEOL 2000FX TEM at 200KV. Aberration-free image can be retrieved by using the proposed oversampling algorithm from the simulated diffraction intensity. Figure 2(a)-(c) show the simulated diffraction intensity of $\mathrm{MgO}$ in [100], [210], and [110] crystallographic orientations. Figure 2(d)-(f) show the results of well-recovered images corresponding to Figures 2 (a)-(c).

After the aberration-free images are retrieved from several diffraction patterns of different crystallographic orientations, three-dimensional structural tomography can be further reconstructed by "Back Projection" algorithm. Electron tomography that gives 3-dimensional microstructure of object has been well developed and widely applied in the biology. Like the oversampling method applied in electron diffraction, the applications of electron tomography in materials science are also in the early stage. The Radon transformation [4] describes the projection through an object by integration with the thickness along the beam direction. Figure 3 shows the three-dimensional structure for the $\mathrm{MgO}$, which is free from aberration.

To be brief, in simulation case we have demonstrated the three-dimensional aberration-free images can be recovered from diffraction patterns of different crystallographic orientations.

\section{Reference}

[1] R.W. Gerchberg and W.O. Saxton., Optik, 35(2):237-246,1972

[2] J. R. Fienup. Optics,21(15):2758-2769, 1982 
[3] J. M. Zuo, I. Vartanyants, Science, vol.300, 2003

[4] H. Furukawa, M. Shimizu, JEOL System Technology Co.,Ltd.

$$
\text { Reciprocal space Real space }
$$
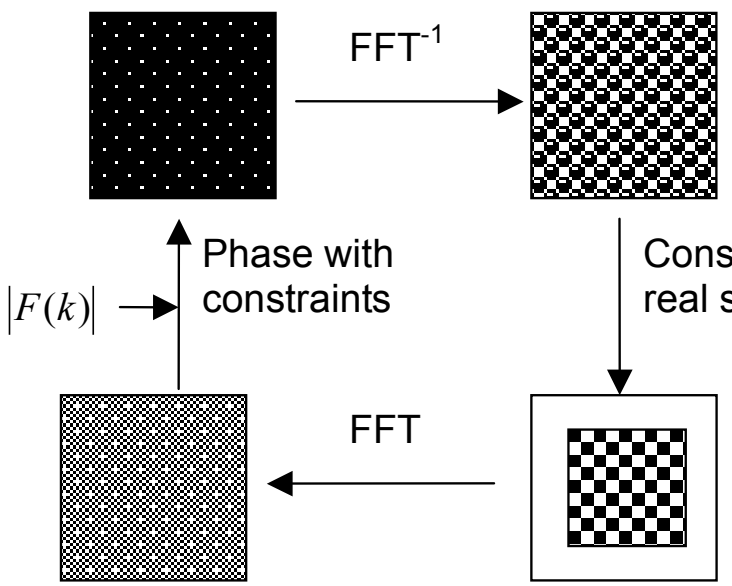

Fig1.The iterative algorithm
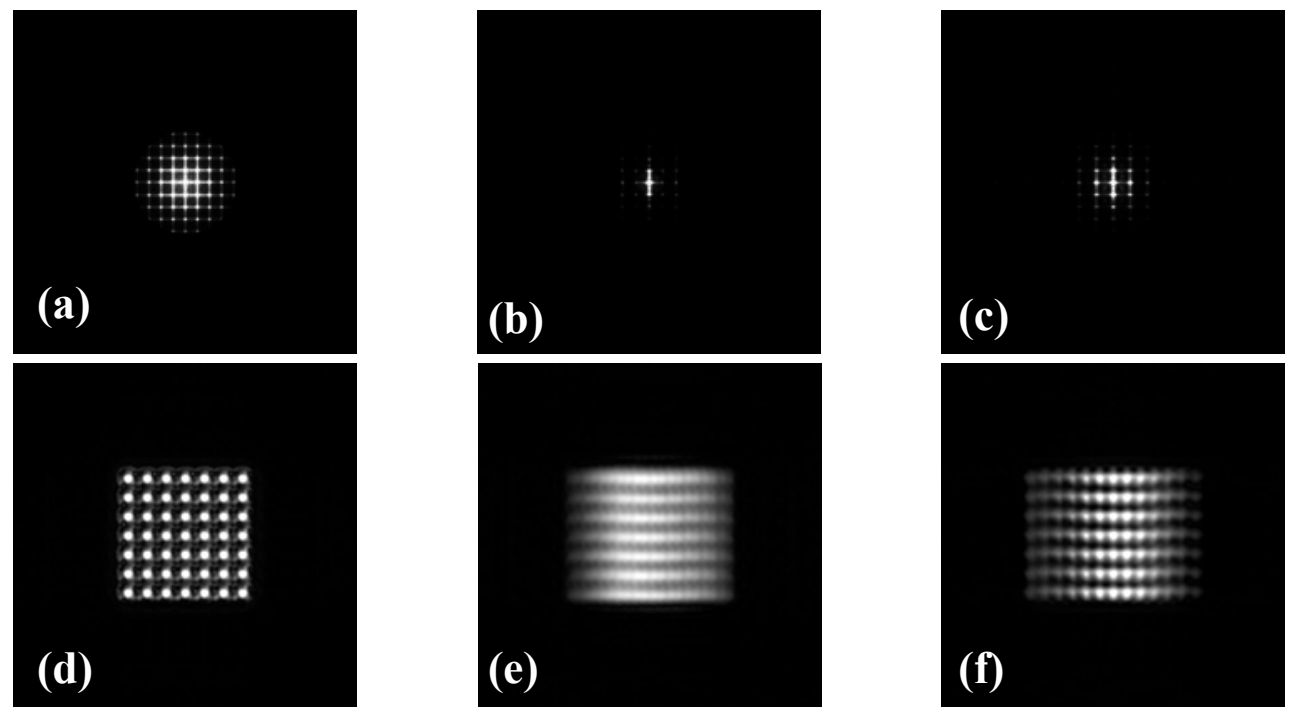

Fig 2.The simulated diffraction pattern (top) in the different directions. Images on the bottom are the results of the recovery by oversampling method.

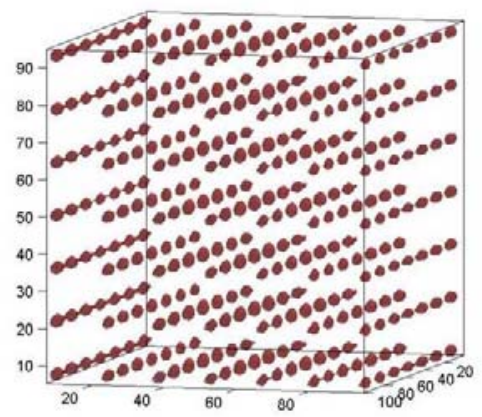

Fig3. The $\mathrm{MgO}(27$ unit cells) reconstructed by diffraction tomography. 